\title{
Generalized Local Homology Modules of Complexes
}

\author{
Fatemeh Mohammadi Aghjeh Mashhad; \\ Islamic Azad University, Parand Branch,Tehran, Iran. \\ Received: 11 Nov. 2014 \\ Revised: 1 July 2017
}

\begin{abstract}
The theory of local homology modules was initiated by Matlis in 1974. It is a dual version of the theory of local cohomology modules. Mohammadi and Divaani-Aazar studied the connection between local homology and Gorenstein flat modules by using Gorenstein flat resolutions. In this paper, we introduce generalized local homology modules for complexes and we give several ways for computing these modules by using Gorenstein flat resolutions. We also find a lower bound for vanishing of generalized local homology modules over a commutative Noetherian ring and we give an upper bound for vanishing of these modules over a commutative Noetherian ring possessing a dualizing complex.
\end{abstract}

Keywords: a-adic completion functor, Generalized local homology modules, Gorenstein flat modules, Gorenstein flat dimension.

2010 Mathematics Subject Classification. 13D05, $13 \mathrm{D} 25$.

\section{Introduction and Prerequisites}

Throughout this paper $\mathrm{R}$ is a commutative Noetherian ring. We recall some definitions from [2] and [6]. A complex $\mathrm{X}$ is a sequence of R-modules $\mathrm{X}_{\mathrm{n}}$ and R-homomorphisms $\partial_{\mathrm{n}}^{\mathrm{X}}$

$$
\mathrm{X}=\cdots \rightarrow \mathrm{X}_{\mathrm{n}+1} \stackrel{\partial_{\mathrm{n}+1}^{\mathrm{X}}}{\rightarrow} \mathrm{X}_{\mathrm{n}} \stackrel{\partial_{\mathrm{n}}^{\mathrm{X}}}{\rightarrow} \mathrm{X}_{\mathrm{n}-1} \rightarrow \cdots,
$$

such that $\partial_{n}^{X} \partial_{n+1}^{X}=0$ for all $n \in Z$. A complex $X$ is said to be bounded to the left (resp. right), if there is an integer $u$ such that $X_{n}=0$ for all $n>u$ (resp. $n<u$ ). An R-module $M$ is thought of as the complex

$$
\cdots \rightarrow 0 \rightarrow \mathrm{M} \rightarrow 0 \rightarrow \cdots
$$

with $\mathrm{M}$ in degree zero. We denote the derived category of R-modules by $\mathrm{D}(\mathrm{R})$ and we use the symbol $\simeq$ for denoting isomorphisms in this category. The supremum and infimum of a complex $X$ in $D(R)$ are defined respectively as $\sup X:=\sup \left\{i \in Z \mid H_{i}(X) \neq 0\right\}$ and $\inf X:=\inf \left\{i \in Z \mid H_{i}(X) \neq 0\right\}$, with the usual conventions that $\sup \phi=-\infty$ and inf $\phi=\infty$. mohammadi_fh@yahoo.com 
A complex $\mathrm{X}$ is said to be non-homologically trivial when its homology complex is not zero. The full subcategory of complexes homologically bounded to the right (resp. left) is denoted by $D_{\sqsupset}(R)$ (resp. $D_{\sqsubset}(R)$ ). Also, the full subcategory of homologically bounded complexes is denoted by $D_{\square}(R)$ and we denote the full subcategory of homologically bounded complexes with finitely generated homology modules by $D_{\square}^{f}(R)$. For any complex $X$ in $D_{\sqsupset}(R)$ (resp. $D_{\sqsubset}(R)$ ), there is a bounded to the right (resp. left) complex $\mathrm{P}$ (resp. I) consisting of projective (resp. injective) R-modules which is isomorphic to $\mathrm{X}$ in $\mathrm{D}(\mathrm{R})$ by $[4$, Theorem A.3.2 P\&F) and I)]. Such a complex $\mathrm{P}$ (resp. I) is called a projective (resp. injective) resolution of $\mathrm{X}$. A complex $\mathrm{X}$ is said to have finite projective (resp. injective) dimension, if $\mathrm{X}$ possesses a bounded projective (resp. injective) resolution. Similarly, a complex $\mathrm{X}$ is said to have finite flat dimension if it is isomorphic (in $\mathrm{D}(\mathrm{R})$ ) to a bounded complex of flat $\mathrm{R}$-modules.

The left derived tensor product functor $-\otimes_{\mathrm{R}}^{\mathrm{L}} \backsim$ is computed by taking a projective resolution of the first argument or of the second one. The right derived homomorphism functor $\operatorname{RHom}_{R}(-, \sim)$ is computed by taking a projective resolution of the first argument or by taking an injective resolution of the second one.

We recall some definitions from the theory of Gorenstein homological dimensions from the text book [5]. An R-module $\mathrm{N}$ is said to be Gorenstein injective if there exists an exact complex I of injective R-modules such that $N \cong i m\left(I_{1} \rightarrow I_{0}\right)$ and $\operatorname{Hom}_{\mathrm{R}}(\mathrm{E}, \mathrm{I})$ is exact for all injective R-modules E. Also, an R-module $\mathrm{M}$ is said to be Gorenstein flat if there exists an exact complex $\mathrm{F}$ of flat $\mathrm{R}$-modules such that $M \cong \operatorname{im}\left(F_{0} \rightarrow F_{-1}\right)$ and $F \otimes_{R} I$ is exact for all injective R-modules $I$. The Gorenstein flat dimension of any complex $\mathrm{X}$ in $\mathrm{D}_{\sqsupset}(\mathrm{R})$ is defined by

$$
\begin{aligned}
& \operatorname{Gfd}_{R} X:=\inf \left\{\sup \left\{1 \in Z \mid Q_{1} \neq 0\right\} \mid Q\right. \text { is a bounded to the right } \\
& \text { complex of Gorenstein flat } R-\text { modules such that } Q \simeq X\} .
\end{aligned}
$$

Moreover a dualizing complex for $\mathrm{R}$ is a complex $\mathrm{D} \in \mathrm{D}_{\square}^{\mathrm{f}}(\mathrm{R})$ such that the homothety morphism, $R \rightarrow R_{H o m}(D, D)$ is an isomorphism in $D(R)$ and $D$ has finite injective dimension.

Let a be an ideal of $\mathrm{R}$. There are two important functors which are effective tools for mathematician working in the theory of commutative algebra and algebraic geometry. One of them is the a-torsion functor $\Gamma_{\mathrm{a}}$ and the other one is the a-adic completion 
functor $\Lambda^{\mathrm{a}}$. We recall that for any R-module $\mathrm{M}$,

$$
\Gamma_{a}(M)=\lim _{\rightarrow_{n}} \operatorname{Hom}_{R}\left(\frac{R}{a^{n}}, M\right), \Lambda^{a}(M)=\lim _{\leftarrow_{n}}\left(\frac{R}{a^{n}} \otimes_{R} M\right) .
$$

One can easily check that the section functor is also an additive left exact functor on the category of complexes of R-modules. The right derived functor of a-torsion functor exists in $D_{\sqsubset}(R)$. For $X \in D_{\sqsubset}(R)$, the complex $R \Gamma_{a}(X) \in D_{\sqsubset}(R)$ is defined by $\mathrm{R} \Gamma_{\mathrm{a}}(\mathrm{X}):=\Gamma_{\mathrm{a}}(\mathrm{I})$, where $\mathrm{I}$ is an (any) injective resolution of $\mathrm{X}$; see [6], [10] and [16] for more details. Also, for any integer $\mathrm{i}$, the i-th local cohomology module of $\mathrm{X}$ with respect to $\mathrm{a}$ is defined by $\mathrm{H}_{\mathrm{a}}^{\mathrm{i}}(\mathrm{X}):=H_{-i}\left(\mathrm{R} \Gamma_{\mathrm{a}}(\mathrm{X})\right)$. Yassemi [16] defined the generalized section functor in the natural manner. For any two complexes $X \in D_{\sqsupset}(R)$ and $Y \in D_{\sqsubset}(R)$, he defined $\mathrm{R} \Gamma_{\mathrm{a}}(\mathrm{X}, \mathrm{Y})$ by $\mathrm{R} \Gamma_{\mathrm{a}}(\mathrm{X}, \mathrm{Y}):=\mathrm{R} \Gamma_{\mathrm{a}}\left(\mathrm{RHom}_{\mathrm{R}}(\mathrm{X}, \mathrm{Y})\right)$ and the $\mathrm{i}$-th generalized local cohomology module of $X$ and $Y$ with respect to a by $H_{a}^{i}(X, Y):=H_{-i}\left(R \Gamma_{a}(X, Y)\right)$ for any integer $i$.

In this paper, we study the generalized local homology modules which are in some sense dual to generalized local cohomology modules and are in fact a generalization of the usual local homology modules. More precisely, we know that the a-adic completion functor defines an additive functor on the category of complexes of R-modules. The left derived functor of a-adic completion functor exists in $D_{\sqsupset}(R)$. For $X \in D_{\sqsupset}(R)$, the complex $\mathrm{L} \Lambda^{\mathrm{a}}(\mathrm{X}) \in \mathrm{D}_{\sqsupset}(\mathrm{R})$ is defined by $\mathrm{L} \Lambda^{\mathrm{a}}(\mathrm{X}):=\Lambda^{\mathrm{a}}(\mathrm{P})$, where $\mathrm{P}$ is a (any) projective resolution of $X$; see [8], [10], and [15] for more details. Also, for any integer $\mathrm{i}$, the $i$-th local homology module of $X$ with respect to a is defined by $H_{i}^{a}(X):=H_{i}\left(L \Lambda^{a}(X)\right)$. For any two complexes $\mathrm{X}, \mathrm{Y} \in \mathrm{D}_{\sqsupset}(\mathrm{R})$, we denote the generalized a-adic completion functor of $\mathrm{X}$ and $\mathrm{Y}$ by $\mathrm{L} \Lambda^{\mathrm{a}}(\mathrm{X}, \mathrm{Y})$ and we define it as $\mathrm{L} \Lambda^{\mathrm{a}}(\mathrm{X}, \mathrm{Y}):=\mathrm{L} \Lambda^{\mathrm{a}}\left(\mathrm{X} \otimes_{\mathrm{R}}^{\mathrm{L}} \mathrm{Y}\right)$. For any integer $i$, we define the $i$-th generalized local homology module of $X$ and $Y$ with respect to a by $H_{i}^{a}(X, Y):=H_{i}\left(L \Lambda^{a}(X, Y)\right)$. Since $L \Lambda^{a}(R,-)=L \Lambda^{a}(-)$, then the generalized a-adic completion functor extends the usual a-adic completion functor. Let $\mathrm{M}$ and $\mathrm{N}$ be two R-modules. The notion of generalized local homology modules

$$
H_{i}^{a}(M, N)=\lim _{t} \operatorname{Tor}_{i}^{R}\left(\frac{M}{a^{t} M}, N\right)
$$

was introduced by Nam in [13, Definition 2.1] and he showed that if $M$ is a finitely generated R-module and $\mathrm{N}$ is a linearly compact $\mathrm{R}$-module, then $\mathrm{H}_{\mathrm{i}}^{\mathrm{a}}(\mathrm{M}, \mathrm{N}) \cong \mathrm{H}_{\mathrm{i}}\left(\mathrm{L} \Lambda^{\mathrm{a}}(\mathrm{M}, \mathrm{N})\right)$ for any integer i, see [14, Theorem 3.6]. 
Since the a-adic completion functor is neither right nor left exact, computing local homology modules and generalized local homology modules is, in general, difficult to do. It is therefore natural and an interesting and challenging problem to try to find a way to compute these modules. Matlis [11] studied local homology modules with respect to ideals of a local ring which are generated by regular sequences. Simon [15] extended the study of local homology modules for any ideal of arbitrary Noetherian rings. Later, Greenlees and May [8] gave a new way for exhibiting local homology modules. Finally, Lipman et al. [10] showed that the local homology modules can be computed by using Cech complexes. Also, Mohammadi and Divaani-Aazar showed that the local homology modules can be computed by Gorenstein flat resolutions, see [12, Theorem 2.5].

In this paper, we extend the results of [12] in order to find new ways for computing generalized local homology modules by using Gorenstein flat resolutions.

Let $\mathrm{X}$ be a non-homologically trivial complex in $\mathrm{D}_{コ}(\mathrm{R})$ and $\mathrm{Q}$ be a bounded to the right complex of Gorenstein flat $\mathrm{R}$-modules such that $\mathrm{Q} \simeq \mathrm{X}$. Let $\mathrm{i}$ be an integer. We prove that:

i. For a non-homologically trivial complex $\mathrm{Y}$ in $\mathrm{D}_{\sqsupset}(\mathrm{R})$ with flat resolution $\mathrm{F}$, we have $\mathrm{H}_{\mathrm{i}}^{\mathrm{a}}(\mathrm{X}, \mathrm{Y}) \cong H_{i}\left(\Lambda^{\mathrm{a}}\left(\mathrm{Q} \otimes_{\mathrm{R}} \mathrm{F}\right)\right)$.

ii. If $R$ possesses a dualizing complex, $X \in D_{\square}(R)$ and $M$ is a nonzero finitely generated R-module with finite flat dimension, then $H_{i}^{a}(M, X) \cong H_{i}\left(M \otimes_{R} \Lambda^{a}(Q)\right)$.

Part i) provides a way to achieve the difficult task of computing generalized local homology modules. Furthermore, it yields the main result of [12] by setting $X:=R$. As for part ii), we actually prove a partial extension of this statement for R-modules without considering existence of dualizing complex and, using this result, we prove that for any $\mathrm{R}$-module $\mathrm{T}, \mathrm{H}_{\mathrm{i}}^{\mathrm{a}}(\mathrm{M}, \mathrm{T})=0$ for all $\mathrm{i}>\mathrm{Gfd}_{\mathrm{R}} \mathrm{T}$, which provides an upper bound for vanishing of generalized local homology modules and moreover improves [12, Lemma 2.2 i)]. We end this paper by giving some bounds for vanishing of generalized local homology modules.

\section{Results}

We start this section by the following lemma which asserts that every Gorenstein flat R-module is $\Lambda$-acyclic for any ideal a of $\mathrm{R}$. We recall that for an ideal a of $\mathrm{R}$ and an R-module $N$, if $H_{i}^{a}(N)=0$ for any positive integer $i$, then $N$ is $\Lambda^{a}$-acyclic. 
Lemma 1. Let a be an ideal of $\mathrm{R}$ and $\mathrm{N}$ a Gorenstein flat $\mathrm{R}$-module. Then $\mathrm{N}$ is $\Lambda^{\mathrm{a}}$-acyclic and there is a natural isomorphism $\mathrm{H}_{0}^{\mathrm{a}}(\mathrm{N}) \cong \Lambda^{\mathrm{a}}(\mathrm{N})$. Moreover, if $\mathrm{R}$ possesses a dualizing complex, then $\Lambda^{\mathrm{a}}(\mathrm{N})$ is Gorenstein flat.

Proof. See [12, Lemmas 2.2 and 2.7].

Theorem 2. Let a be an ideal of $R$. Let $X \in D_{\sqsupset}(R)$ and $Q$ be a bounded to the right complex of Gorenstein flat $R$-modules such that $Q \simeq X$. Then $L \Lambda^{\mathrm{a}}(\mathrm{X}) \simeq \Lambda^{\mathrm{a}}(\mathrm{Q})$, and so $H_{i}^{a}(X)=H_{i}\left(\Lambda^{a}(Q)\right)$ for all $i \in Z$. In particular, sup $L \Lambda^{a}(X) \leq \operatorname{Gfd}_{R} X$. Moreover, if $R$ possesses a dualizing complex, then $\operatorname{Gfd}_{R} L \Lambda^{a}(X) \leq \operatorname{Gfd}_{R} X$.

Proof. See [12, Theorem 2.5 and Corollary2.8 i)].

For proving our main result, we need the following proposition.

Proposition 3. The following assertions hold:

i. The class of Gorenstein flat R-modules is closed under direct sums.

ii. If $\mathrm{M}$ is a Gorenstein flat $\mathrm{R}$-module and $\mathrm{N}$ is a flat $\mathrm{R}$-module, then $\mathrm{M} \otimes_{\mathrm{R}} \mathrm{N}$ is a Gorenstein flat R-module.

iii. Assume that $\mathrm{R}$ possesses a dualizing complex. If $\mathrm{M}$ is a Gorenstein injective R-module and $\mathrm{N}$ is an injective R-module, then $\operatorname{Hom}_{\mathrm{R}}(\mathrm{M}, \mathrm{N})$ is a Gorenstein flat R-module.

Proof. See [9, Proposition 3.2] and [3, Ascent table I a) and i)].

In the first two parts of the following theorem, we give several ways to compute generalized local homology modules of complexes.

Theorem 4. Let a be an ideal of $\mathrm{R}$ and $\mathrm{X}, \mathrm{Y} \in \mathrm{D}_{\sqsupset}(\mathrm{R})$ be two non-homologically trivial complexes. The following assertions hold for any integer i:

i. If $\mathrm{Q}$ is a bounded to the right complex of Gorenstein flat R-modules such that $\mathrm{Q} \simeq \mathrm{X}$ and $\mathrm{F}$ is a flat resolution of $\mathrm{Y}$, then $\mathrm{L} \Lambda^{\mathrm{a}}\left(\mathrm{X} \otimes_{\mathrm{R}}^{\mathrm{L}} \mathrm{Y}\right) \simeq \Lambda^{\mathrm{a}}\left(\mathrm{Q} \otimes_{\mathrm{R}} \mathrm{F}\right)$, and so $\mathrm{H}_{\mathrm{i}}^{\mathrm{a}}(\mathrm{X}, \mathrm{Y}) \cong \mathrm{H}_{\mathrm{i}}\left(\Lambda^{\mathrm{a}}\left(\mathrm{Q} \otimes_{\mathrm{R}} \mathrm{F}\right)\right)$.

Moreover, if $\mathrm{R}$ possesses a dualizing complex, then the following hold:

ii. Assume that $\mathrm{M}$ is a nonzero finitely generated $\mathrm{R}$-module such that either it is flat or injective dimension is finite. If $\mathrm{F}$ is a bounded to the right complex of Gorenstein flat $R$-modules such that $F \simeq Y$ and $Y \in D_{\square}(R)$, then $\mathrm{L} \Lambda^{\mathrm{a}}\left(\mathrm{M} \otimes_{\mathrm{R}}^{\mathrm{L}} \mathrm{Y}\right) \simeq \mathrm{M} \otimes_{\mathrm{R}} \Lambda^{\mathrm{a}}(\mathrm{F})$, and so $\quad \mathrm{H}_{\mathrm{i}}^{\mathrm{a}}(\mathrm{M}, \mathrm{Y}) \cong \mathrm{H}_{\mathrm{i}}\left(\mathrm{M} \otimes_{\mathrm{R}} \Lambda^{\mathrm{a}}(\mathrm{F})\right)$. 
iii. Assume that $X, Y \in D_{\square}(R)$. If $E$ is a bounded to the left complex of Gorenstein injective $\mathrm{R}$-modules such that $\mathrm{X} \simeq \mathrm{E}$ and $\mathrm{I}$ is a bounded injective resolution of $\mathrm{Y}$, then $L \Lambda^{a}\left(R \operatorname{Hom}_{R}(X, Y)\right) \simeq \Lambda^{a}\left(\operatorname{Hom}_{R}(E, I)\right)$, and so

$$
\mathrm{H}_{\mathrm{i}}^{\mathrm{a}}\left(\mathrm{R} \operatorname{Hom}_{\mathrm{R}}(\mathrm{X}, \mathrm{Y})\right) \cong \mathrm{H}_{\mathrm{i}}\left(\Lambda^{\mathrm{a}}\left(\operatorname{Hom}_{\mathrm{R}}(\mathrm{E}, \mathrm{I})\right)\right) \text {. }
$$

\section{Proof.}

i. Note that $\left(\mathrm{Q} \otimes_{\mathrm{R}} \mathrm{F}\right)_{\mathrm{i}}=\bigsqcup_{\mathrm{p} \in Z} \mathrm{Q}_{\mathrm{p}} \otimes_{\mathrm{R}} \mathrm{F}_{\mathrm{i}-\mathrm{p}}$ for any $\mathrm{i} \in \mathrm{Z}$. Then, by Proposition 3 i) and ii), every component of $\mathrm{Q} \otimes_{R} F$ is Gorenstein flat $\mathrm{R}$-module. Therefore $\mathrm{Q} \otimes_{R} F$ is a bounded to the right complex of Gorenstein flat $\mathrm{R}$-modules such that $\mathrm{Q} \otimes_{\mathrm{R}} \mathrm{F} \simeq \mathrm{X} \otimes_{\mathrm{R}}^{\mathrm{L}} \mathrm{Y}$. This implies that $\mathrm{L} \Lambda^{\mathrm{a}}\left(\mathrm{X} \otimes_{\mathrm{R}}^{\mathrm{L}} \mathrm{Y}\right) \simeq \Lambda^{\mathrm{a}}\left(\mathrm{Q} \otimes_{\mathrm{R}} \mathrm{F}\right)$ by Theorem 2 .

Now, assume that $\mathrm{R}$ possesses a dualizing complex.

ii. By Lemma 1, every component of $\Lambda^{\mathrm{a}}(\mathrm{F})$ is Gorenstein flat R-module and by Theorem 2, $\mathrm{L} \Lambda^{\mathrm{a}}(\mathrm{Y}) \simeq \Lambda^{\mathrm{a}}(\mathrm{F})$. Now by using [12, Lemma 2.6] and [4,orollary 2.16], we have:

$$
\begin{gathered}
\left(M \otimes_{\mathrm{R}}^{\mathrm{L}} \mathrm{Y}\right) \simeq \mathrm{M} \otimes_{\mathrm{R}}^{\mathrm{L}} \mathrm{L} \Lambda^{\mathrm{a}}(\mathrm{Y}) \\
\simeq \mathrm{M} \otimes_{\mathrm{R}}^{\mathrm{L}} \Lambda^{\mathrm{a}}(\mathrm{F}) \\
\simeq \mathrm{M} \otimes_{\mathrm{R}} \Lambda^{\mathrm{a}}(\mathrm{F}) .
\end{gathered}
$$

iii. Note that $\operatorname{Hom}_{R}(X, Y) \in D_{\sqsupset}(R)$, because

$$
\inf R \operatorname{Hom}_{R}(X, Y) \geq-i d_{R} Y-\operatorname{supX}
$$

by [2, Corollary A.5.2] and also by $[6,6.15]$

$$
\mathrm{RHom}_{\mathrm{R}}(\mathrm{X}, \mathrm{Y}) \simeq \operatorname{Hom}_{\mathrm{R}}(\mathrm{X}, \mathrm{I}) \simeq \operatorname{Hom}_{\mathrm{R}}(\mathrm{E}, \mathrm{I}) .
$$

Set $\mathrm{s}:=\min \left\{\mathrm{i} \in \mathrm{Z} \mid \mathrm{I}_{\mathrm{i}} \neq 0\right\}, \mathrm{u}:=\max \left\{\mathrm{i} \in \mathrm{Z} \mid \mathrm{I}_{\mathrm{i}} \neq 0\right\}$ and $\mathrm{z}:=\max \left\{\mathrm{i} \in \mathrm{Z} \mid \mathrm{E}_{\mathrm{i}} \neq 0\right\}$. Then for any integer $\mathrm{i}$, we have

$$
\operatorname{Hom}_{\mathrm{R}}(\mathrm{E}, \mathrm{I})_{\mathrm{i}}=\bigoplus_{\mathrm{t}=\mathrm{s}}^{\mathrm{t}=\mathrm{u}} \operatorname{Hom}_{\mathrm{R}}\left(\mathrm{E}_{\mathrm{t}-\mathrm{i}}, \mathrm{I}_{\mathrm{t}}\right),
$$

and also if $\mathrm{i}<\mathrm{s}-\mathrm{z}$, then $\operatorname{Hom}_{\mathrm{R}}(\mathrm{E}, \mathrm{I})_{\mathrm{i}}=0$. So, by Proposition $\left.3 \mathrm{i}\right)$ and iii), $\operatorname{Hom}_{R}(\mathrm{E}, \mathrm{I})$ is a bounded to the right complex of Gorenstein flat R-modules which implies that $L \Lambda^{\mathrm{a}}\left(\operatorname{RHom}_{\mathrm{R}}(\mathrm{X}, \mathrm{Y})\right) \simeq \Lambda^{\mathrm{a}}\left(\operatorname{Hom}_{\mathrm{R}}(\mathrm{E}, \mathrm{I})\right)$ by Theorem 2 .

We need the following lemma for finding another way to compute generalized local homology modules.

Lemma 5. Let a be an ideal of $R$ and $M$ a finitely generated $R$-module with finite flat dimension. Assume that $\mathrm{N}$ is an R-module and $\mathrm{F}=\cdots \rightarrow \mathrm{F}_{2} \rightarrow \mathrm{F}_{1} \rightarrow \mathrm{F}_{0} \rightarrow 0$, is a bounded to the right complex of Gorenstein flat R-modules such that $F \simeq N$. Set $T:=M \otimes_{R} H_{0}^{a}(-)$. 
Then for any integer i, we have

$$
\mathrm{L}_{\mathrm{i}} \mathrm{T}(\mathrm{N}) \cong \mathrm{H}_{\mathrm{i}}\left(\mathrm{M} \otimes_{\mathrm{R}} \mathrm{H}_{0}^{\mathrm{a}}(\mathrm{F})\right),
$$

where $\mathrm{H}_{0}^{\mathrm{a}}(\mathrm{F})=\cdots \rightarrow \mathrm{H}_{0}^{\mathrm{a}}\left(\mathrm{F}_{2}\right) \rightarrow \mathrm{H}_{0}^{\mathrm{a}}\left(\mathrm{F}_{1}\right) \rightarrow \mathrm{H}_{0}^{\mathrm{a}}\left(\mathrm{F}_{0}\right) \rightarrow 0$.

Proof. It is easy to see that $\mathrm{T}$ is a right exact covariant functor from the category of R-modules and R-homomorphisms to itself. First, we show that every Gorenstein flat $\mathrm{R}$-module $\mathrm{Q}$ is T-acyclic. Let $\mathrm{P}=\cdots \rightarrow \mathrm{P}_{2} \rightarrow \mathrm{P}_{1} \rightarrow \mathrm{P}_{0} \rightarrow 0$ be a projective resolution of $\mathrm{Q}$, and $\mathrm{i}$ be an integer. We have

$$
\begin{aligned}
& \mathrm{L}_{\mathrm{i}} \mathrm{T}(\mathrm{Q})=\mathrm{H}_{\mathrm{i}}(\mathrm{T}(\mathrm{P})) \\
& =\mathrm{H}_{\mathrm{i}}\left(\mathrm{M} \otimes_{\mathrm{R}} \mathrm{H}_{0}^{\mathrm{a}}(\mathrm{P})\right) \\
& \stackrel{(\mathrm{a})}{\cong} \mathrm{H}_{\mathrm{i}}\left(\mathrm{M} \otimes_{\mathrm{R}} \Lambda^{\mathrm{a}}(\mathrm{P})\right) \\
& \stackrel{(b)}{\cong} \mathrm{H}_{\mathrm{i}}\left(\mathrm{M} \otimes{ }_{\mathrm{R}}^{\mathrm{L}} \Lambda^{\mathrm{a}}(\mathrm{P})\right) \\
& \cong \mathrm{H}_{\mathrm{i}}\left(\mathrm{M} \otimes \mathrm{R}_{\mathrm{L}}^{\mathrm{L}} \Lambda^{\mathrm{a}}(\mathrm{Q})\right) \\
& \stackrel{(\mathrm{c})}{\cong}\left(\mathrm{M} \otimes_{\mathrm{R}}^{\mathrm{L}} \Lambda^{\mathrm{a}}(\mathrm{Q})\right) \\
& =\operatorname{Tor}_{i}^{\mathrm{R}}\left(\mathrm{M}, \Lambda^{\mathrm{a}}(\mathrm{Q})\right) \\
& \stackrel{(d)}{=} 0 \text {, }
\end{aligned}
$$

where (a) follows from the fact that the following two complexes

$$
\mathrm{H}_{0}^{\mathrm{a}}(\mathrm{P})=\cdots \rightarrow \mathrm{H}_{0}^{\mathrm{a}}\left(\mathrm{P}_{2}\right) \rightarrow \mathrm{H}_{0}^{\mathrm{a}}\left(\mathrm{P}_{1}\right) \rightarrow \mathrm{H}_{0}^{\mathrm{a}}\left(\mathrm{P}_{0}\right) \rightarrow 0,
$$

and

$$
\Lambda^{\mathrm{a}}(\mathrm{P})=\cdots \rightarrow \Lambda^{\mathrm{a}}\left(\mathrm{P}_{2}\right) \rightarrow \Lambda^{\mathrm{a}}\left(\mathrm{P}_{1}\right) \rightarrow \Lambda^{\mathrm{a}}\left(\mathrm{P}_{0}\right) \rightarrow 0,
$$

are isomorphisms (see e.g. the proof of [11, Corollary 4.6]), where (b) follows from the fact that the a-adic completion of every flat R-module is flat (see e.g. $[1,1.4 .7]$ ), where (c) is due to Theorem 2, and finally (d) is by [12, Lemma 2.7]. Hence, every component of $\mathrm{F}$ is $\mathrm{T}$-acyclic. This completes the proof. Indeed, recall that if $\mathrm{T}$ is a right exact additive functor from the category of R-modules and R-homomorphisms to itself, then for any R-module $\mathrm{N}$, the left derived functors $\mathrm{L}_{\mathrm{i}} \mathrm{T}$ of $\mathrm{T}$ at $\mathrm{N}$ can be computed by using left resolutions of $\mathrm{N}$ which are consisting of $\mathrm{T}$-acyclic R-modules.

The second part of the following lemma partially improves Theorem 4 ii) without the existence of dualizing complex.

Lemma 6. Let a be an ideal of $\mathrm{R}$ and $\mathrm{M}$ a nonzero finitely generated R-module. Assume that $\mathrm{N}$ is an $\mathrm{R}$-module and $\mathrm{i}$ is an integer. We have:

i) If $N$ is a Gorenstein flat R-module, then $H_{i}^{a}(M, N) \simeq H_{i}\left(M \otimes_{R}^{L} \Lambda^{a}(N)\right)$. 
ii) If $\mathrm{F}=\cdots \rightarrow \mathrm{F}_{2} \rightarrow \mathrm{F}_{1} \rightarrow \mathrm{F}_{0} \rightarrow 0$ is a bounded to the right complex of Gorenstein flat $\mathrm{R}$-modules such that $\mathrm{F} \simeq \mathrm{N}$, and if $\mathrm{M}$ has finite flat dimension, then

$$
\mathrm{H}_{\mathrm{i}}^{\mathrm{a}}(\mathrm{M}, \mathrm{N}) \simeq \mathrm{H}_{\mathrm{i}}\left(\mathrm{M} \otimes_{\mathrm{R}} \Lambda^{\mathrm{a}}(\mathrm{F})\right) .
$$

\section{Proof.}

i) $[12$, Lemma 2.6] and Theorem 2 yield that

$$
\mathrm{L} \Lambda^{\mathrm{a}}\left(\mathrm{M} \otimes_{\mathrm{R}}^{\mathrm{L}} \mathrm{N}\right) \simeq \mathrm{M} \otimes_{\mathrm{R}}^{\mathrm{L}} \mathrm{L} \Lambda^{\mathrm{a}}(\mathrm{N}) \simeq \mathrm{M} \otimes_{\mathrm{R}}^{\mathrm{L}} \Lambda^{\mathrm{a}}(\mathrm{N}),
$$

and so $H_{i}^{a}(M, N) \simeq H_{i}\left(M \otimes_{R}^{L} \Lambda^{a}(N)\right)$ as desired.

ii) Let $\mathrm{P}=\cdots \rightarrow \mathrm{P}_{2} \rightarrow \mathrm{P}_{1} \rightarrow \mathrm{P}_{0} \rightarrow 0$ be a projective resolution of $\mathrm{N}$, and $\mathrm{i}$ be an integer.

We have

$$
\begin{gathered}
\mathrm{H}_{\mathrm{i}}^{\mathrm{a}}(\mathrm{M}, \mathrm{N})=\mathrm{H}_{\mathrm{i}}\left(\mathrm{L} \Lambda^{\mathrm{a}}\left(\mathrm{M} \otimes_{\mathrm{R}}^{\mathrm{L}} \mathrm{N}\right)\right) \\
\stackrel{(\mathrm{a})}{\cong} \mathrm{H}_{\mathrm{i}}\left(\mathrm{M} \otimes_{\mathrm{R}}^{\mathrm{L}} \mathrm{L} \Lambda^{\mathrm{a}}(\mathrm{N})\right) \\
\cong \mathrm{H}_{\mathrm{i}}\left(\mathrm{M} \otimes_{\mathrm{R}}^{\mathrm{L}} \Lambda^{\mathrm{a}}(\mathrm{P})\right) \\
\stackrel{(\mathrm{b})}{\cong} \mathrm{H}_{\mathrm{i}}\left(\mathrm{M} \otimes_{\mathrm{R}} \Lambda^{\mathrm{a}}(\mathrm{P})\right) \\
\stackrel{(\mathrm{c})}{\cong} \mathrm{H}_{\mathrm{i}}\left(\mathrm{M} \otimes_{\mathrm{R}} \mathrm{H}_{0}^{\mathrm{a}}(\mathrm{P})\right) \\
\stackrel{(\mathrm{d})}{\cong} \mathrm{H}_{\mathrm{i}}\left(\mathrm{M} \otimes_{\mathrm{R}} \mathrm{H}_{0}^{\mathrm{a}}(\mathrm{F})\right) \\
\stackrel{(\mathrm{e})}{\cong} \mathrm{H}_{\mathrm{i}}\left(\mathrm{M} \otimes_{\mathrm{R}} \Lambda^{\mathrm{a}}(\mathrm{F})\right),
\end{gathered}
$$

where (a) follows from [12, Lemma 2.6], and where (b) follows from the fact that the a-adic completion of every flat R-module is flat (see e.g. [1, 1.4.7]). Isomorphism (c) follows from the fact that the following two complexes

$$
\Lambda^{\mathrm{a}}(\mathrm{P})=\cdots \rightarrow \Lambda^{\mathrm{a}}\left(\mathrm{P}_{2}\right) \rightarrow \Lambda^{\mathrm{a}}\left(\mathrm{P}_{1}\right) \rightarrow \Lambda^{\mathrm{a}}\left(\mathrm{P}_{0}\right) \rightarrow 0,
$$

and

$$
\mathrm{H}_{0}^{\mathrm{a}}(\mathrm{P})=\cdots \rightarrow \mathrm{H}_{0}^{\mathrm{a}}\left(\mathrm{P}_{2}\right) \rightarrow \mathrm{H}_{0}^{\mathrm{a}}\left(\mathrm{P}_{1}\right) \rightarrow \mathrm{H}_{0}^{\mathrm{a}}\left(\mathrm{P}_{0}\right) \rightarrow 0,
$$

are isomorphism (see e.g. the proof of [11, Corollary 4.6]). Isomorphism (d) follows from Lemma 5. Finally (e) is obtained by using Lemma 1.

The following corollary improves [12, Lemma 2.2 i)].

Corollary 7. Let a be an ideal of $\mathrm{R}, \mathrm{M}$ a nonzero finitely generated R-module with finite flat dimension and $\mathrm{N}$ an $\mathrm{R}$-module. Then $\mathrm{H}_{\mathrm{i}}^{\mathrm{a}}(\mathrm{M}, \mathrm{N})=0$, for all $\mathrm{i}>\operatorname{Gfd}_{\mathrm{R}} \mathrm{N}$. In particular, if $\mathrm{N}$ is Gorenstein flat, then $\mathrm{H}_{\mathrm{i}}^{\mathrm{a}}(\mathrm{M}, \mathrm{N})=0$ for all $\mathrm{i}>0$.

Proof. Let $t:=\operatorname{Gfd}_{R} N<\infty$. Then there is a bounded to the right complex of Gorenstein flat R-modules of the form

$$
\mathrm{F}=0 \rightarrow \mathrm{F}_{\mathrm{t}} \rightarrow \mathrm{F}_{\mathrm{t}-1} \rightarrow \ldots \rightarrow \mathrm{F}_{1} \rightarrow \mathrm{F}_{0} \rightarrow 0,
$$


such that $F \simeq N$. By Lemma 6 ii $), H_{i}^{a}(M, N) \cong H_{i}\left(M \otimes_{R} \Lambda^{a}(F)\right)$ for any integer $i$ and this completes the proof.

We recall that for any ideal a of $\mathrm{R}$ and any complex $\mathrm{X} \in \mathrm{D}_{\sqsupset}(\mathrm{R}), a$-width of $\mathrm{X}$ is defined as:

$$
w i d t h(a, X):=\inf \left(\frac{R}{a} \otimes_{R}^{L} X\right)
$$

We finish this paper by the following proposition which gives lower and upper bounds for vanishing of generalized local homology modules.

Proposition 8. Let a be an ideal of $R$ and $X, Y \in D_{\sqsupset}(R)$. Then

i. $\quad \inf \mathrm{L} \Lambda^{\mathrm{a}}\left(\mathrm{X} \otimes_{\mathrm{R}}^{\mathrm{L}} \mathrm{Y}\right) \geq$ width $_{\mathrm{R}}(\mathrm{a}, \mathrm{X})+\inf \mathrm{Y}$.

ii. Assume that $R$ possesses a dualizing complex and $X \in D_{\square}(R)$. Let $Y \in D_{\square}^{f}(R)$ be a non-homologically trivial complex such that either its projective or injective dimension is finite and $\operatorname{Gfd}_{R} X<\infty$. Then $\sup L \Lambda^{a}\left(X \otimes{ }_{R}^{L} Y\right) \leq \operatorname{dim} R+\sup X+\sup Y$.

\section{Proof.}

i. By [2, Proposition A.4.15], we have $X \otimes_{R}^{L} Y \in D_{\sqsupset}(R)$. Also [7, Theorem 2.11] and [2, A.4.15.1] imply that:

$$
\begin{aligned}
\inf L \Lambda^{\mathrm{a}}( & \left(\mathrm{X} \otimes_{\mathrm{R}}^{\mathrm{L} Y}\right)=\text { width }_{\mathrm{R}}\left(\mathrm{a}, \mathrm{X} \otimes_{\mathrm{R}}^{\mathrm{L}} \mathrm{Y}\right) \\
& =\inf \left(\mathrm{R} / \mathrm{a} \otimes_{\mathrm{R}}^{\mathrm{L}}\left(\mathrm{X} \otimes_{\mathrm{R}}^{\mathrm{L}} \mathrm{Y}\right)\right) \\
& \geq \inf \left(\mathrm{R} / \mathrm{a} \otimes_{\mathrm{R}}^{\mathrm{L}} \mathrm{X}\right)+\inf \mathrm{Y} \\
& =\text { width }_{\mathrm{R}}(\mathrm{a}, \mathrm{X})+\inf \mathrm{Y} .
\end{aligned}
$$

ii. By [12, Corollary 2.8 ii)] and [4, 1.4 and Theorem 3.5], we have

$$
\sup L \Lambda^{\mathrm{a}}\left(\mathrm{X} \otimes_{\mathrm{R}}^{\mathrm{L}} \mathrm{Y}\right) \leq \mathrm{Gfd}_{\mathrm{R}} \mathrm{X}+\sup \mathrm{Y} \leq \operatorname{dim} \mathrm{R}+\sup \mathrm{X}+\sup \mathrm{Y} .
$$

\section{References}

1. Bartijn J., "Flatnes, completions, regular sequences un m’enage`a trios", Thesis, Utrecht (1985).

2. Christensen L. W., "Gorenstein dimensions, Lecture Notes in Mathematics", 1747, Springer-Verlag, Berlin (2000).

3. Christensen L.W., Holm H., "Ascent properties of Auslander categories", Canad. J. Math., 61(1) (2009) 76-108.

4. Christensen L. W., Frankild A., Holm H., "On Gorenstein projective, injective and flat dimensions-a functorial description with applications", J. Algebra, 302(1) (2006) 231-279. 
5. Enoch E. E., Jenda O. M. G., "Relative homological algebra", de Gruyter Expositions in Mathematics, 30, Walter de Gruyter \& Co., Berlin (2000).

6. Foxby H-B., "Hyperhomological algebra \& commutative rings", in preparation.

7. Frankild A., "Vanishing of local homology", Math. Z., 244 (2003) 615-630.

8. Greenlees J. P. C., May J. P., "Derived functors of the $I$-adic completion and local homology", J. Algebra, 149 (1992) 438-453.

9. Holm H., "Gorenstein homological dimensions", J. Pure Appl. Algebra, 189 (2004) 167-193.

10. Lipman J., L'opez A. J., Tarrio L. A., "Local homology and cohomology on schemes", Ann. Sci. Ecole Norm. Sup. , 30(4) (1997) 1-39.

11. Matlis E., "The Koszul complex and duality", Comm. Algebra, 1(2) (1974) 87-144.

12. Mohammadi Aghjeh Mashhad F., Divaani-Aazar K., "local homology and Gorenstein flat modules", Journal of Algebra and its Applications, 11(2) (2012) 12500221-12500228.

13. Nam T. T., "Generalized local homology modules for artinian modules", Algebra Colloquium, 19(1) (2012) 1205-1212.

14. Nam T. T., "Left derived functors of the generalized I-adic completion and generalized local homology", comm. In Algebra, 38 (2010) 440-453.

15. Simon A-M., "Some homological properties of complete modules", Math. Proc. Cambridge Philos. Soc., 108 (2) (1990) 231-246.

16. Yassemi S.,"Generalized section functors", J. Pure Appl. Algebra, 95(1) (1994) 103-119. 